\title{
On the Monodromy Conjecture for curves on normal surfaces
}

\author{
B. Rodrigues*
}

September 3, 2003

\begin{abstract}
This paper is devoted to some results about the Monodromy Conjecture. The conjecture states that for a nonconstant regular function $f$ on a surface germ $(S, 0)$ with $f(0)=0$ we have that if a rational number $s_{\circ}$ is a pole of the topological zeta function $Z_{\text {top }}(f, s)$, then $e^{2 \pi i s}$ is an eigenvalue of the local monodromy of $f$ at some point of $f^{-1}\{0\}$. First we give our own, very elementary and conceptual, proof of the conjecture for the well-known case that the germ $(S, 0)$ is nonsingular. This proof is not only included for its simplicity, but also because we will need exactly the same arguments in the second part. There we will explain what can be expected in the singular case and what certainly not.
\end{abstract}

\section{$1 \quad$ Introduction and preliminaries}

(1.1) Let $(S, 0)$ be a normal complex (algebraic) surface germ and $f$ a nonconstant regular function on $(S, 0)$. For simplicity of notation we assume that $f(0)=0$. In what follows we will often suppose, without explicitly mentioning it anymore, that such a surface germ $(S, 0)$ and a regular function $f$ are given.

We call a morphism $h: X \rightarrow S$ an embedded resolution of $f^{-1}\{0\}$ in $(S, 0)$ if $X$ is nonsingular, $h$ is a proper birational morphism, the restriction $h: X \backslash h^{-1}\{0\} \rightarrow$ $S \backslash\{0\}$ is an isomorphism and the divisor $h^{-1}\left(f^{-1}\{0\}\right)$ has nonsingular irreducible components which intersect transversely.

Recall that there exists a unique minimal embedded resolution, in the sense that any other embedded resolution factors through it by blowing up a finite number of points.

(1.2) We denote by $E_{i}, i \in T=T_{e} \cup T_{s}$, the irreducible components of $h^{-1}\left(f^{-1}\{0\}\right)$, where $T_{e}$ ranges over the exceptional curves and $T_{s}$ over the components of the strict

${ }^{*}$ Research Assistant of the Belgian Fund for Scientific Research - Flanders.

2000 Mathematics Subject Classifficaton. 14B05 14E15 14J17 32S40 
transform of $f^{-1}\{0\}$. For $i \in T_{e}$ we also set

$$
E_{i}^{\circ}:=E_{i} \backslash \bigcup_{\substack{j \in T \\ j \neq i}} E_{j}
$$

Now we define the numerical data $\left(\nu_{i}, N_{i}\right)$ of the curves $E_{i}, i \in T$. First denote by $N_{i}$ the multiplicity of $E_{i}$ in the divisor of $f \circ h$ and for $i \in T_{s}$ put $\nu_{i}=1$. For $i \in T_{e}$ we let $\nu_{i}$ be the $\log$ discrepancy of $E_{i}$. These rational numbers are defined by the equation

$$
K_{X}=h^{*} K_{S}+\sum_{i \in T_{e}}\left(\nu_{i}-1\right) E_{i}
$$

where for a normal variety $V$ we denote by $K_{V}$ its canonical divisor (class). Recall that the pullback $h^{*} A$ of a divisor $A$ on $S$ was first defined by Mumford in [9]. Briefly spoken, $h^{*} A$ is by definition equal to $\widetilde{A}+\sum_{i \in T_{e}} a_{i} E_{i}$, where $\widetilde{A}$ is the strict transform of $A$ and the $a_{i}$ are the unique solutions of the linear system of equations $\left(\widetilde{A}+\sum_{i \in T_{e}} a_{i} E_{i}\right) \cdot E_{k}=0, k \in T_{e}$, using that the intersection matrix of the curves $E_{i}, i \in T_{e}$, is negative definite.

(1.3) Finally we are ready to define the topological zeta function. Let $(S, 0)$ be a normal surface germ and $f$ a regular function on $S$, which of course is nonconstant and satisfies $f(0)=0$ as assumed in (1.1). Let $h: X \rightarrow S$ be any embedded resolution of $f^{-1}\{0\}$ in $(S, 0)$. The topological zeta function associated to $(S, 0)$ and $f$ is defined as

$$
Z_{\mathrm{top}}(f, s):=\sum_{i \in T_{e}} \frac{\chi\left(E_{i}^{\circ}\right)}{\nu_{i}+s N_{i}}+\sum_{\{i, j\} \subseteq T} \frac{\chi\left(E_{i} \cap E_{j}\right)}{\left(\nu_{i}+s N_{i}\right)\left(\nu_{j}+s N_{j}\right)},
$$

where $\chi(\cdot)$ denotes the topological Euler-Poincaré characteristic. Remark that the curves $E_{i}, i \in T_{e}$, can have arbitrary genus $g_{i}$; so $\chi\left(E_{i}^{\circ}\right)=2-2 g_{i}-\operatorname{card}\left(E_{i} \cap\right.$ $\left.\left(\cup_{j \neq i} E_{j}\right)\right)$.

The results of this paper will also apply to the motivic zeta function. Before we pass to its definition we give a brief description of the Grothendieck ring of complex varieties. In this context a variety is not necessarily irreducible, it is a reduced separated scheme of finite type over $\mathbb{C}$. We denote by $K_{0}\left(\operatorname{Var}_{\mathbb{C}}\right)$ the Grothendieck group (or ring) of complex varieties. It is the abelian group generated by the symbols $[X]$, for $X$ a variety, with the relations $[X]=[Y]$ if $X$ and $Y$ are isomorphic as varieties, and $[X]=[Y]+[X \backslash Y]$ if $Y$ is Zariski closed in $X$. There is a natural ring structure on $K_{0}\left(\operatorname{Var}_{\mathbb{C}}\right)$, the product of $[X]$ and $[Y]$ being equal to $\left[X \times_{\mathbb{C}} Y\right]$. We set $\mathbb{L}:=\left[\mathbb{A}_{\mathbb{C}}^{1}\right]$ and denote by $\mathcal{M}_{\mathbb{C}}$ the ring obtained from $K_{0}\left(\operatorname{Var}_{\mathbb{C}}\right)$ by inverting $\mathbb{L}$.

The motivic zeta function associated to $(S, 0)$ and $f$ is defined as

$$
Z_{\text {mot }}(f, s):=\sum_{i \in T_{e}}\left[E_{i}^{\circ}\right] \frac{\mathbb{L}-1}{\mathbb{L}^{\nu_{i}+s N_{i}}-1}+\sum_{\{i, j\} \subseteq T}\left[E_{i} \cap E_{j}\right] \frac{(\mathbb{L}-1)^{2}}{\left(\mathbb{L}^{\nu_{i}+s N_{i}}-1\right)\left(\mathbb{L}^{\nu_{j}+s N_{j}}-1\right)},
$$


where [•] denotes the class in $\mathcal{M}_{\mathbb{C}}$ and where the expression $\mathbb{L}^{-s}$ should be considered as a variable.

These zeta functions on normal surface germs are first introduced by Veys in [13]. He verifies there that the defining expressions are independent of the chosen resolution, and he explains in detail in which ring the motivic zeta function lives, see [13, Subsection 5.2]. With these functions he generalized the topological and motivic zeta function associated to a nonconstant regular function on a nonsingular variety (of arbitrary dimension), which go back to Denef and Loeser, see [5] and [6].

(1.4) We are especially interested in the poles of $Z_{\text {top }}(f, s)$ and $Z_{\text {mot }}(f, s)$. For the topological zeta function the notion of a pole is immediately clear; for the motivic zeta function we use the definition of a pole introduced in [11]. Very briefly, we just have to represent $Z_{\text {mot }}(f, s)$ as a quotient of two polynomials in the variable $\mathbb{L}^{-s}$ over a "well-chosen" ring $A$, say for example $Z_{\text {mot }}(f, s)=N\left(\mathbb{L}^{-s}\right) / D\left(\mathbb{L}^{-s}\right)$. The main point about $A$ is that in this ring the elements $1-\mathbb{L}^{q}$ and $b \mathbb{L}^{a}$, for $q \in \mathbb{Q} \backslash\{0\}, b \in \mathbb{Z} \backslash\{0\}$ and $a \in \mathbb{Q}$, are units; see [11, Subsection 4.4] for the rigorous definition of $A$. Now we simply say that a rational number $q$ is a pole of $Z_{\text {mot }}(f, s)$ if there exists a number $m \in \mathbb{N} \backslash\{0\}$ such that $\left(\mathbb{L}^{-s}-\mathbb{L}^{-q}\right)^{m} \mid D\left(\mathbb{L}^{-s}\right)$ in $A\left[\mathbb{L}^{-s}\right]$, but $\left(\mathbb{L}^{-s}-\mathbb{L}^{-q}\right)^{m} \nmid N\left(\mathbb{L}^{-s}\right)$ in $A\left[\mathbb{L}^{-s}\right]$.

In the case of a regular function on a nonsingular variety of arbitrary dimension $n$, the poles are the subject of the remarkable Monodromy Conjecture [5, 6], which relates them to eigenvalues of the local monodromy of $f: Y \rightarrow \mathbb{C}$, and which is still open for $n \geq 3$. For the definition of local monodromy, see (1.5).

(1.4.1) Monodromy Conjecture. If $s_{\circ}$ is a pole of $Z_{\mathrm{top}}(f, s)$, then $e^{2 \pi i s_{\circ}}$ is an eigenvalue of the local monodromy of $f$ at some point of $f^{-1}\{0\}$.

The formulation of the conjecture for $Z_{\text {mot }}(f, s)$ is completely analogous. When $S=\mathbb{A}^{2}$ and $f$ is a polynomial in two variables, this conjecture has already been proved by Loeser [7]. In fact, Loeser proved the analogous statement for Igusa's local zeta function, from which the stated result follows, see also Remark 2.4(ii). Recall that Igusa originally conjectured the general assertion on $\mathbb{A}^{n}$ for his local zeta function. In [10] this conjecture is already proved for $n=3$ under the extra condition that $f$ is an homogeneous polynomial satisfying $\chi\left(\mathbb{P}_{\mathbb{C}}^{2} \backslash\{f=0\}\right) \neq 0$, and in [2] it is proved for some more surface singularities.

Note that each component $E_{i}, i \in T$, induces a candidate pole $s_{\circ}=-\frac{\nu_{i}}{N_{i}}$ for the topological zeta function $Z_{\text {top }}(f, s)$. However, due to miraculous cancellations, usually many of these candidates are no pole. This strange phenomenon would be elucidated by the Monodromy Conjecture.

Since the above statement of the Monodromy Conjecture certainly does not hold for regular functions on arbitrary normal surfaces, see for example [13, Example 2.8], Veys proposed in [13, Subsection 2.9] to investigate the following adapted version.

Let $c$ denote the least common denominator of the $\log$ discrepancies $\nu_{i}$ and let $s_{\circ}$ 
be a pole of $Z_{\mathrm{top}}(f, s)$. Is then $e^{2 \pi i c s o}$ an eigenvalue of the local monodromy of $f$ at some point of $f^{-1}\{0\}$ ?

We will prove that this "generalized" question indeed has a positive answer when $c=1$ and $s_{\circ} \leq 0$; remark that this includes the case of nonsingular surfaces, which was originally proved by Loeser [7]. On the other hand, when $c=1$ and $s_{\circ}>0$, we will give a considerable indication that the proposed adaptation does not hold anymore. Finally, when $c>1$, we will give an actual counterexample, providing a negative answer to the question of Veys.

(1.5) Fix a normal surface germ $(S, 0)$ and a nonconstant regular function $f$ on it, satisfying $f(0)=0$. We now briefly sketch the definition of local monodromy. Note that exactly the same definition applies to the case of a regular function on a variety germ of arbitrary dimension.

Fix $b \in S$ with $f(b)=0$. Suppose that $(S, b)$ is embedded in $\left(\mathbb{C}^{N}, b\right)$, for some sufficiently large $N \in \mathbb{N}$. Let $B \subseteq \mathbb{C}^{N}$ be a small enough ball with center $b$; the restriction $f_{\mid(B \cap S)}$ is a topological fibration over a small enough pointed disc $D \subseteq \mathbb{C} \backslash\{0\}$ with center 0 . The fiber $M_{(f, b)}$ of this fibration is called the Milnor fiber. The counterclockwise generator of the fundamental group of $D$ induces an endomorphism of $H^{\cdot}\left(M_{(f, b)}, \mathbb{C}\right)$ which is called the local monodromy of $f$ at $b$. By an eigenvalue of the local monodromy of $f$ at $b$ we mean an eigenvalue of the monodromy action on (at least) one of the $H^{q}\left(M_{(f, b)}, \mathbb{C}\right)$ for $0 \leq q \leq \operatorname{dim} S-1=1$.

We will only need the following formula (of A'Campo) in terms of an embedded resolution $h: X \rightarrow S$ of $f^{-1}\{0\}$ in $(S, 0)$.

(1.5.1) Theorem ([1, Théorème 3] and [12, Theorem II.1.3]). For $b \in f^{-1}\{0\}$ let $P_{q}(t)$ denote the characteristic polynomial of the monodromy action on $H^{q}\left(M_{(f, b)}, \mathbb{C}\right)$ for $q \in\{0,1\}$. Then

$$
\frac{P_{1}(t)}{P_{0}(t)}=\prod_{i \in T}\left(1-t^{N_{i}}\right)^{-\chi\left(E_{i}^{\circ} \cap h^{-1}\{b\}\right)} .
$$

Note that a similar formula always exists for a variety germ $(V, 0)$ and a regular function $f$ on it whenever the singular locus $V_{\text {sing }}$ of $V$ is contained in $f^{-1}\{0\}$; see [12, Theorem II.1.3].

\section{The special case of a nonsingular germ $(S, 0)$}

(2.1) We will give here our own and very elementary proof of the well-known case of Conjecture 1.4.1 that $(S, 0)$ is a nonsingular surface germ. We will use the following lemma.

(2.2) Lemma. Let $E_{1}, \ldots, E_{r}$ be nonsingular, complete curves on a nonsingular 
surface, such that $\bigcup_{i=1}^{r} E_{i}$ is connected and has only normal crossings. For each $i \in\{1, \ldots, r\}$ we put $E_{i}^{\circ}:=E_{i} \backslash \bigcup_{j \neq i} E_{j}$. Then we have

$$
\sum_{i=1}^{r} \chi\left(E_{i}^{\circ}\right) \leq 0,
$$

except when $\bigcup_{i=1}^{r} E_{i}$ is a tree consisting of rational curves. Moreover, in the latter case we have $\sum_{i=1}^{\bar{r}} \chi\left(E_{i}^{\circ}\right)=2$.

Proof. First suppose that at least one of the curves $E_{i}$ is non-rational, say for example $E_{1}$. We will use some induction argument on $r$. When $r=1$, we obviously have

$$
\chi\left(E_{1}^{\circ}\right)=\chi\left(E_{1}\right)=2-2 g_{1} \leq 0,
$$

where $g_{1}$ denotes the genus of $E_{1}$. For arbitrary $r$, we can use the induction hypothesis on the $r-1$ first curves, yielding that

$$
\sum_{i=1}^{r-1} \chi\left(\widetilde{E}_{i}\right) \leq 0,
$$

where $\widetilde{E}_{i}$ stands for

$$
E_{i} \backslash \bigcup_{\substack{j=1 \\ j \neq i}}^{r-1} E_{j}
$$

Clearly $\chi\left(E_{i}^{\circ}\right) \leq \chi\left(\widetilde{E}_{i}\right)$ for $i \in\{1, \ldots, r-1\}$. Since $\bigcup_{i=1}^{r} E_{i}$ is connected, we see that $\chi\left(E_{r}^{\circ}\right) \leq 1$ and that there exists at least one $i \in\{1, \ldots, r-1\}$ satisfying $\chi\left(E_{i}^{\circ}\right) \leq \chi\left(\widetilde{E}_{i}\right)-1$. Then we have

$$
\begin{aligned}
\sum_{i=1}^{r} \chi\left(E_{i}^{\circ}\right) & \leq \chi\left(E_{r}^{\circ}\right)-1+\sum_{i=1}^{r-1} \chi\left(\widetilde{E}_{i}\right) \\
& \leq 0 .
\end{aligned}
$$

Now suppose that all curves $E_{i}$ are rational. Again, building up the configuration $\bigcup_{i=1}^{r} E_{i}$ by introducing one by one the curves $E_{i}$, we obtain that always $\sum_{i=1}^{r} \chi\left(E_{i}^{\circ}\right) \leq 2$ and that $\sum_{i=1}^{r} \chi\left(E_{i}^{\circ}\right) \leq 0$ as soon as there is a cycle of rational curves in the configuration. On the other hand, when $\bigcup_{i=1}^{r} E_{i}$ is a tree consisting of rational curves, the same induction argument will easily reveal that $\sum_{i=1}^{r} \chi\left(E_{i}^{\circ}\right)=2$.

(2.3) Proof of Conjecture 1.4.1. We use the notation of Section 1 for the minimal embedded resolution $h: X \rightarrow S$ of $f^{-1}\{0\}$ in $(S, 0)$. Suppose that $s_{\circ} \in \mathbb{Q}$ is a pole of $Z_{\text {top }}(f, s)$. Then we know by [11, Theorem 2.8(i)] that either $s_{\circ}=-\frac{1}{N_{i}}$ for some irreducible component $E_{i}$ of the strict transform of $f^{-1}\{0\}$ (Case 1), or $s_{\circ}=-\frac{\nu_{i}}{N_{i}}$ for some (rational) exceptional curve $E_{i}$ intersecting at least three times other components (Case 2). The first case is easy. Indeed, let $b \in S$ be any point, 
different from 0 , on the irreducible component of $f^{-1}\{0\}$ whose strict transform by $h$ is precisely $E_{i}$. Theorem 1.5.1 for example then yields that the zeros of

$$
1-t^{N_{i}}
$$

are eigenvalues of the local monodromy of $f$ at $b$, implying that $e^{2 \pi i s_{\circ}}=e^{2 \pi i\left(-\frac{1}{N_{i}}\right)}$ is such a monodromy eigenvalue.

Now we suppose that Case 2 is satisfied, but Case 1 is not. Denote by $E_{i_{\circ}}$ the curve postulated in Case 2, and consider the formula of A'Campo for $b=0$, stating that the alternating product of the characteristic polynomials of the local monodromy of $f$ at 0 is equal to

$$
\prod_{i \in T_{e}}\left(1-t^{N_{i}}\right)^{-\chi\left(E_{i}^{\circ}\right)}
$$

Write

$$
s_{\circ}=-\frac{a}{d},
$$

where $a$ and $d$ are two natural numbers which are relatively prime. So we are obviously especially interested in the curves $E_{i}$ which satisfy $d \mid N_{i}$. We may as well assume that $d \nmid N_{i}$ for $i \in T_{s}$, since for the other possibility we can use a very similar treatment as for Case 1.

Clearly it is sufficient to prove that

$$
\sum_{\substack{i \in T_{e} \\ d \mid N_{i}}} \chi\left(E_{i}^{\circ}\right)<0 .
$$

First note that each connected component of $N:=\bigcup_{i \in T_{e}, d \mid N_{i}} E_{i}$ contains at least one curve intersecting at least two times other components $E_{j}$ with $d \nmid N_{j}$. Here we use the well-known relation $\kappa N=\sum_{i=1}^{r} N_{i}$, where $E$ is an exceptional curve intersecting $r$ times other components $E_{i}$, for $i=1, \ldots, r$, and where $-\kappa$ denotes the self-intersection number of $E$. This relation can easily be deduced by intersecting the principal divisor $\sum_{i \in T} N_{i} E_{i}$ with $E$; see also [13, Lemma 2.3]. By Lemma 2.2 we obtain that

$$
\sum_{i \in I} \chi\left(E_{i}^{\circ}\right) \leq 0
$$

for every connected component $\bigcup_{i \in I} E_{i}$ of $N$, the equality only occurring when $\bigcup_{i \in I} E_{i}$ is a tree (consisting of rational curves and) intersecting $\bigcup_{j \in T, d \nmid N_{j}} E_{j}$ exactly twice. We will now show that for the connected component $M=\bigcup_{i \in I_{M}} E_{i}$ of $N$ containing $E_{i}$ 。 we certainly have

$$
\sum_{i \in I_{M}} \chi\left(E_{i}^{\circ}\right)<0
$$

finishing the proof of Conjecture 1.4.1.

Suppose that $\sum_{i \in I_{M}} \chi\left(E_{i}^{\circ}\right)=0$, or equivalently that $M$ is a tree (consisting of rational curves and) intersecting $\bigcup_{j \in T, d \nmid N_{j}} E_{j}$ exactly twice. Obviously those two intersecting points have to belong to the same curve $E_{1}$ of $M$. We distinguish two 
possibilities. First, suppose that $E_{i_{\circ}}=E_{1}$. Then there still is at least one component $E_{2}$ of $M$ intersecting $E_{i_{\circ}}$. By [13, Lemma 2.3(iii)] we know that

$$
\sum_{i=1}^{k} \alpha_{i}=k-2,
$$

where the summation runs over all curves $E_{i}$ intersecting $E_{i_{\circ}}$, the notation $\alpha_{i}$ stands for $\nu_{i}+s_{\circ} N_{i}$ and $k \geq 3$ denotes the number of curves intersecting $E_{i_{\circ}}$. We also know by [11, Lemma 2.6] that all $\alpha_{i}<1$. The combination of those two facts easily results in the inequality $\alpha_{i}>-1$. Since moreover

$$
\alpha_{2}=\nu_{2}+s_{\circ} N_{2}=\nu_{2}-\frac{a}{d} N_{2} \in \mathbb{Z},
$$

we obtain that $\alpha_{2}=0$ and hence that

$$
s_{\circ}=-\frac{\nu_{2}}{N_{2}} .
$$

With similar arguments we see that $E_{2}$ also has to intersect another component of $M$, say $E_{3}$, and that in addition

$$
-\frac{\nu_{2}}{N_{2}}=-\frac{\nu_{3}}{N_{3}}
$$

Repeating the same procedure seems to produce an infinite chain, which of course contradicts the finiteness of the resolution graph.

Now suppose that $E_{i_{\circ}} \neq E_{1}$. Then there are at least three components of $M$ intersecting $E_{i_{\circ}}$. For each of them we can start the same procedure as above. Since there is only one curve in $M$ which intersects components $E_{j}$ with $d \nmid N_{j}$, we obviously end up with the same contradiction.

\section{(2.4) Remarks.}

(i) In exactly the same way one can prove Conjecture 1.4.1 for the motivic zeta function $Z_{\text {mot }}(f, s)$.

(ii) Let $f \in F\left[x_{1}, x_{2}\right] \backslash F$ for some (big enough) number field. To any $p$-adic completion $K$ of $F$ one associates Igusa's local zeta function $Z^{K}(f, s)$, which is the meromorphic continuation to $\mathbb{C}$ of

$$
s \longmapsto \int_{P_{K}^{2}}|f(x)|^{s}|d x|,
$$

for $\Re(s)>0$, where $P_{K}$ denotes the maximal ideal of the valuation ring $R_{K}$ of $K$. For some more detailed explanation about this definition (and also for the formula of Denef writing $Z^{K}(f, s)$ in terms of an embedded resolution), we refer to [3], [4] or [10, Introduction and Section 1]. 
Again we consider the minimal embedded resolution. Recall the result of Loeser [7] stating that if $s_{\circ}$ is a pole of $Z^{K}(f, s)$, then $\Re\left(s_{\circ}\right)=-\frac{1}{N_{i}}$ for some irreducible component $E_{i}$ of the strict transform of $f^{-1}\{0\}$ or $\Re\left(s_{\circ}\right)=-\frac{\nu_{i}}{N_{i}}$ for some exceptional curve $E_{i}$ intersecting at least three times other components. For almost all completions $K$ of $F$ (i.e., for all except a finite number) one can also easily check this by hand using the formula for $Z^{K}(f, s)$ in terms of an embedded resolution. So we can use exactly the same arguments as in the previous proof to obtain the correctness of the following (original) $p$-adic version of the Monodromy Conjecture.

For almost all completions $K$ of $F$, if $s_{\circ}$ is a pole of $Z^{K}(f, s)$, then $e^{2 \pi i \Re\left(s_{\circ}\right)}$ is an eigenvalue of the local monodromy of $f$ at some complex point of $f^{-1}\{0\}$.

\section{The general case}

(3.1) In this section we will see what happens when we proceed to the case of normal surface germs $(S, 0)$. Veys already showed [13, Example 2.8] that the assertion in (1.4.1) is in general not true for singular surfaces $S$. In [13, Subsection 2.9] he proposes to investigate the following adaptation of the Monodromy Conjecture for normal surface germs. Let $c$ denote the least common denominator of the $\log$ discrepancies $\nu_{i}, i \in T_{e}$; it is independent of the chosen resolution. Let $s_{\circ}$ be a pole of $Z_{\text {top }}(f, s)$; is then $e^{2 \pi i c s 。}$ an eigenvalue of the local monodromy of $f$ at some point of $f^{-1}\{0\}$ ?

Here we will mainly work on this question of Veys. We will first consider the situation that all the log discrepancies are actually integral, including of course the Gorenstein singularities. Obviously this condition is independent of the chosen resolution. Note that in this case the above integer $c$ is equal to 1 and we find ourselves with the usual formulation of the Monodromy Conjecture. We have the following affirmative answer for the nonpositive poles of $Z_{\text {top }}(f, s)$.

(3.2) Theorem. Let $f$ be a nonconstant regular function on a normal surface germ $(S, 0)$ with $f(0)=0$ and suppose that all log discrepancies are integral. Let $s_{\circ} \in \mathbb{Q}_{<0}$. If $s_{\circ}$ is a pole of $Z_{\text {top }}(f, s)$, then $e^{2 \pi i s_{\circ}}$ is an eigenvalue of the local monodromy of $f$ at some point of $f^{-1}\{0\}$.

Remark. The same theorem (and proof) will hold for $Z_{\operatorname{mot}}(f, s)$.

Proof. We use the notation of Section 1 for the minimal embedded resolution $h$ : $X \rightarrow S$ of $f^{-1}\{0\}$ in $(S, 0)$. First remark that $e^{2 \pi i 0}=1$ is always an eigenvalue of the local monodromy of $f$ at any point $b$ of $f^{-1}\{0\}$ different from 0 .

For the negative poles we can copy word for word the argument in the proof of Conjecture 1.4.1, see (2.3). 
(3.3) Bearing in mind the details of the proof of Conjecture 1.4.1 (and of Theorem 3.2) we are led to introduce the following definition. Fix an arbitrary embedded resolution $h: X \rightarrow S$ of $f^{-1}\{0\}$ in $(S, 0)$ and let $d \in \mathbb{N} \backslash\{0\}$. We will say that the pair $(f ;(S, 0))$ is bad for $d$ when

(i) $d \nmid N_{i}$ for $i \in T_{s}$,

(ii) all connected components $\bigcup_{i \in I} E_{i}$ of $\bigcup_{i \in T_{e}, d \mid N_{i}} E_{i}$ are trees consisting of rational curves and

(iii) each of these trees $\bigcup_{i \in I} E_{i}$ intersects $\bigcup_{j \in T, d \nmid N_{j}} E_{j}$ exactly twice.

One easily verifies that this condition is independent of the chosen resolution. Note moreover that in condition (iii) the two intersection points necessarily have to belong to the same curve of the tree $\bigcup_{i \in I} E_{i}$.

(3.4) Let us now look at the most general case of an arbitrary nonconstant regular function $f$ on an arbitrary normal surface germ $(S, 0)$ with $f(0)=0$ and an arbitrary rational number $s_{\circ}$. Write $s_{\circ}=a / d$ for some numbers $a \in \mathbb{Z}$ and $d \in \mathbb{N} \backslash\{0\}$ which are relatively prime. We can repeat more or less the previous proof up to the situation that $d \nmid N_{i}$ for $i \in T_{s}$ and that we still have to prove that at least one of the connected components $\bigcup_{i \in I} E_{i}$ of $N:=\bigcup_{i \in T_{e}, d \mid N_{i}} E_{i}$ satisfies

$$
\sum_{i \in I} \chi\left(E_{i}^{\circ}\right)<0
$$

Note that by Lemma 2.2 we already know that $\sum_{i \in I} \chi\left(E_{i}^{\circ}\right) \leq 0$ for every connected component of $N$. At this stage the point is not only that we cannot follow anymore the details of the proof of Conjecture 1.4.1 (and of Theorem 3.2), but also that we will be able to show that when $s_{\circ}>0$ or $c>1$ such a desired connected component of $N$ does not need to exist, see Example 3.5 and Example 3.6. Obviously, imposing the negation of the above "geometric" badness condition immediately results in the following proposition.

(3.4.1) Proposition. Let $f$ be a nonconstant regular function on a normal surface germ $(S, 0)$ with $f(0)=0$. Let $s_{\circ} \in \mathbb{Q}$ and write $s_{\circ}=a / d$ for some numbers $a \in \mathbb{Z}$ and $d \in \mathbb{N} \backslash\{0\}$ which are relatively prime. Suppose that the pair $(f ;(S, 0))$ is not bad for $d$. Then we have the following.

If $s_{\circ}$ is a pole of $Z_{\mathrm{top}}(f, s)$, then $e^{2 \pi i s}$ is an eigenvalue of the local monodromy of $f$ at some point of $f^{-1}\{0\}$.

Exactly the same comments (and examples) apply to the question of Veys involving the least common denominator $c$ of the $\log$ discrepancies $\nu_{i}, i \in T_{e}$. Here the statement is as follows.

(3.4.2) Proposition. Let $f$ be a nonconstant regular function on a normal surface germ $(S, 0)$ with $f(0)=0$ and denote by $c$ the least common denominator of the $\log$ 
discrepancies $\nu_{i}, i \in T_{e}$. Let $s_{\circ} \in \mathbb{Q}$ and write $c s_{\circ}=a / d$ for some numbers $a \in \mathbb{Z}$ and $d \in \mathbb{N} \backslash\{0\}$ which are relatively prime. Suppose that the pair $(f ;(S, 0))$ is not bad for $d$. Then we have the following.

If $s_{\circ}$ is a pole of $Z_{\text {top }}(f, s)$, then $e^{2 \pi i c s}$ is an eigenvalue of the local monodromy of $f$ at some point of $f^{-1}\{0\}$.

Remark. The same propositions hold for $Z_{\text {mot }}(f, s)$.

(3.5) Example. We will give here a considerable indication that in Proposition 3.4.1 and Proposition 3.4.2 the condition on the pair $(f ;(S, 0))$ of being not bad can not be omitted, providing a negative answer to the question of Veys in [13, Subsection 2.9]. This first example will provide a situation with $c=1$ (meaning that all $\log$ discrepancies are integral) and $s_{\circ}>0$. Note that in this case both propositions actually coincide.

We use the following combination of a result of Wall [14, Theorem 2.1] and Manetti [8, Theorem 3.2], see also [11, Subsection 2.13] for some more details.

Let $\left\{g_{i} \mid 1 \leq i \leq n\right\},\left\{b_{i} \mid 1 \leq i \leq n\right\}$ and $\left\{b_{i, j} \mid 1 \leq i, j \leq n\right\}$ be three sets of integers such that $g_{i}, b_{i} \geq 0$ for each $i, b_{j, i}=b_{i, j} \geq 0$ for each $i \neq j$ and the quadratic form $\sum_{i, j=1}^{n} b_{i, j} x_{i} x_{j}$ is negative definite. Then there exists an isolated algebraic surface singularity $(S, 0)$, an algebraic curve germ $C$ on $S$ and an embedded resolution $h: X \rightarrow S$ of $C$ in $(S, 0)$ with exceptional divisor $D=\bigcup_{i=1}^{n} E_{i}$ such that $g_{i}$ is the genus of $E_{i}, b_{i}=E \cdot E_{i}$ and $b_{i, j}=E_{i} \cdot E_{j}$, where $E$ denotes the strict transform of $C$ by $h$.

The singularity is normal if and only if $D$ is connected.

In this example we put $n=6, g_{1}=g_{2}=g_{3}=g_{4}=g_{5}=g_{6}=0, b_{1}=b_{2}=b_{3}=b_{4}=$ $b_{5}=0, b_{6}=1$ and

$$
B=\left(\begin{array}{cccccc}
-4 & 0 & 1 & 0 & 0 & 0 \\
0 & -2 & 1 & 0 & 0 & 0 \\
1 & 1 & -1 & 1 & 0 & 0 \\
0 & 0 & 1 & -6 & 1 & 1 \\
0 & 0 & 0 & 1 & -3 & 0 \\
0 & 0 & 0 & 1 & 0 & -2
\end{array}\right)
$$

Then there exists a normal surface germ $(S, 0)$, a curve germ $C$ on $S$ and an embedded resolution $h: X \rightarrow S$ of $C$ in $(S, 0)$ realizing six exceptional curves $E_{i}$, $i=1, \ldots, 6$, equipped with the specified data. Moreover, the strict transform $E$ of $C$, which we consider this time with $(1,7)$ as numerical data, is a prime divisor on $X$ satisfying $E \cdot E_{1}=E \cdot E_{2}=E \cdot E_{3}=E \cdot E_{4}=E \cdot E_{5}=0$ and $E \cdot E_{6}=1$. Then the embedded resolution graph has the following form: 


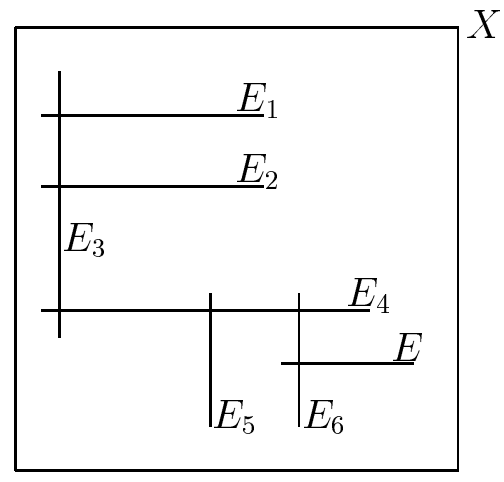

Figure 1

Solve the well-known equations [13, Lemma 2.3] for the numerical data. This yields

$$
\begin{gathered}
\nu_{1}=-1, \nu_{2}=-2, \nu_{3}=-5, \nu_{4}=-1, \nu_{5}=\nu_{6}=0 \\
N_{1}=3, N_{2}=6, N_{3}=12, N_{4}=3, N_{5}=1, N_{6}=5
\end{gathered}
$$

Consequently

$$
Z_{\text {top }}(f, s)=\frac{-24-35 s+273 s^{2}}{(-1+3 s)(-5+12 s)(1+7 s)},
$$

implying that $s_{\circ}=-\frac{\nu_{4}}{N_{4}}=\frac{1}{3}$ is a pole (of order 1) of $Z_{\mathrm{top}}(f, s)$. Nevertheless, the complex number $e^{2 \pi i s_{\circ}}$ is neither a zero nor a pole of one of the following expressions :

$$
\begin{gathered}
\frac{1}{1-t^{7}}, \\
\prod_{i=1}^{6}\left(1-t^{N_{i}}\right)^{-\chi\left(E_{i}^{\circ}\right)}=\frac{\left(1-t^{12}\right)\left(1-t^{3}\right)}{\left(1-t^{3}\right)\left(1-t^{6}\right)(1-t)}=\frac{1+t^{6}}{1-t} .
\end{gathered}
$$

The reason that at the beginning of this example we only talked about a considerable indication and not about an actual counterexample, is that we do not know whether or not there exists a regular function on $(S, 0)$ realizing the curve germ $C$ on $S$ (together with its numerical data). But if there exists such a regular function $f$, this example provides a topological zeta function having $s_{\circ}=\frac{1}{3}$ as a pole without $e^{2 \pi i s_{\circ}}$ being an eigenvalue of the local monodromy of $f$.

(3.6) Example. We end this chapter with an example in which the least common denominator $c$ of the $\log$ discrepancies is not equal to 1 . We proceed completely in the same way as in the previous example, but this time we put $n=5, g_{1}=g_{2}=$ $g_{3}=g_{4}=g_{5}=0$ and

$$
B=\left(\begin{array}{ccccc}
-2 & 0 & 1 & 0 & 0 \\
0 & -2 & 1 & 0 & 0 \\
1 & 1 & -3 & 1 & 0 \\
0 & 0 & 1 & -2 & 1 \\
0 & 0 & 0 & 1 & -6
\end{array}\right)
$$


The prime divisor $E$ on $X$ has to satisfy $E \cdot E_{1}=E \cdot E_{2}=E \cdot E_{3}=E \cdot E_{5}=0$ and $E \cdot E_{4}=1$, and we associate to it the numerical data $(1,16)$. This yields an embedded resolution graph of the following form:

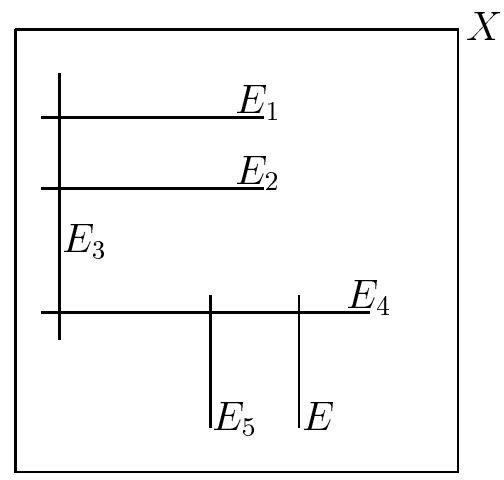

Figure 2

The numerical data are

$$
\begin{gathered}
\nu_{1}=\nu_{2}=\frac{17}{32}, \quad \nu_{3}=\frac{1}{16}, \nu_{4}=\frac{1}{8}, \nu_{5}=\frac{3}{16} \\
N_{1}=N_{2}=3, N_{3}=6, N_{4}=12, \quad N_{5}=2 .
\end{gathered}
$$

Hence the least common denominator $c$ of the rational numbers $\nu_{i}$ is 32 . Also remark that the singularity $(S, 0)$ is log terminal. Since

$$
\frac{\nu_{3}}{N_{3}}=\frac{\nu_{4}}{N_{4}}=\frac{1}{96}
$$

we know that $s_{\circ}=-\frac{1}{96}$ is a pole (of order 2) of $Z_{\text {top }}(f, s)$. Nevertheless, the complex number $e^{2 \pi i c s 。}=e^{2 \pi i(-1 / 3)}$ is neither a zero nor a pole of one of the following expressions :

$$
\begin{gathered}
\frac{1}{1-t^{16}} \\
\prod_{i=1}^{5}\left(1-t^{N_{i}}\right)^{-\chi\left(E_{i}^{\circ}\right)}=\frac{\left(1-t^{6}\right)\left(1-t^{12}\right)}{\left(1-t^{3}\right)^{2}\left(1-t^{2}\right)}=\frac{\left(1+t^{3}\right)^{2}\left(1+t^{6}\right)}{1-t^{2}} .
\end{gathered}
$$

Since $(S, 0)$ is a rational singularity, there certainly exists a regular function $f$ on $(S, 0)$ realizing the divisor $16 C$, where again $C:=h(E)$. Hence we have a topological zeta function $Z_{\text {top }}(f, s)$ with $s_{\circ}=-\frac{1}{96}$ as a pole, but without $e^{2 \pi i c s 。}$ being an eigenvalue of the local monodromy of $f$. Again this shows the necessity of the condition on $(f ;(S, 0))$ in Proposition 3.4 .2 of being not bad. The same note also applies to Proposition 3.4.1, checking this time that $e^{2 \pi i s_{\circ}}=e^{2 \pi i(-1 / 96)}$ is not an eigenvalue of the local monodromy of $f$.

\section{References}

[1] N. A'Campo, La fonction zeta d'une monodromie, Comment. Math. Helv. 50 (1975), 233-248. 
[2] E. Artal, P. Cassou-Noguès, I. Luengo, and A. Melle, Monodromy Conjecture for some surface singularities, Ann. Sci. École Norm. Sup. (2002).

[3] J. Denef, Local zeta function and Euler characteristics, Duke Math. J. 63 (1991), 713-721.

[4] _ Report on Igusa's local zeta function, Sém. Bourbaki 201/202/203 (1991), 359-386.

[5] J. Denef and F. Loeser, Caractéristiques d'Euler-Poincaré, fonctions zeta locales, et modifications analytiques, J. Amer. Math. Soc. 5 (1992), 705-720.

[6] _ Motivic Igusa zeta functions, J. Algebraic Geom. 7 (1998), 505-537.

[7] F. Loeser, Fonctions d'Igusa p-adiques et polynômes de Bernstein, Amer. J. Math. 110 (1988), 1-22.

[8] M. Manetti, Algebraization of analytic curve germs passing through isolated surface singularities, Arch. Math. 66 (1996), 428-440.

[9] D. Mumford, The topology of normal singularities of an algebraic surface and a criterion for simplicity, Publ. Math. I.H.E.S. 9 (1961), 5-22.

[10] B. Rodrigues and W. Veys, Holomorphy of Igusa's and topological zeta functions for homogeneous polynomials, Pacific J. Math. 201 No. 2 (2001), 429-440.

[11] _ Poles of zeta functions on normal surfaces, Proc. London Math. Soc., to appear (2002), 35p.

[12] M. Tibăr, The Lefschetz Number of a Monodromy Transformation, Ph. D. thesis, Univ. Utrecht, 1992.

[13] W. Veys, The topological zeta function associated to a function on a normal surface germ, Topology 38 (1999), 439-456.

[14] C.T.C. Wall, Quadratic forms and normal surface singularities, In Quadratic forms and their applications, Contemporary Math. 272 (Amer. Math. Soc., 2000), 293-311.

K.U.LEUVEN, Departement Wiskunde, Celestijnenlaan 200B, 3001 LEUVEN, BELGIUM

E-mail address: bart.rodrigues@wis.kuleuven.ac.be 